\title{
Un ponte culturale tra memoria e storia: la biografia di comunità
}

L'articolo presenta un progetto di ricerca che si prefigge di ricomporre la dicotomia tra storia e memoria coniugando una tecnica di raccolta delle biografie con lo studio di luoghi e comunità di particolare rilevanza storico-culturale (ad esempio luoghi di stragi ed eccidi). L'obiettivo è ricostruire il profilo di una comunità tramite una narrazione autobiografica collettiva e la sua trasmissione intergenerazionale.

This article presents a research project that aims to reconcile the dichotomy between history and memory, combining a technique of collection of the biographies with the study of sites and communities of particular historical and cultural significance (as places of war-massacres). The goal is to rebuild the profile of a community through a collective autobiographical narrative and its intergenerational transmission.

\section{Introduzione}

Progettare una "biografia di comunità" significa ricomporre la dicotomia tra memoria e storia nell'ambito di un orizzonte di ricerca che coniughi le tecniche di raccolta di biografie con lo studio di luoghi e comunità di particolare rilevanza storico-culturale. Ad esempio, luoghi che sono stati lo scenario di stragi e/o eccidi bellici, oppure comunità che rivestono un particolare valore per le espressioni culturali, linguistiche o sociali che le caratterizzano. L'analisi e la rielaborazione dei racconti biografici ci può dimostrare se, come e in che misura quell'evento sia stato elaborato, trasmesso o non trasmesso, se sia diventato coscienza della comunità, o sia invece rimasto circoscritto ad alcuni protagonisti, senza trasformarsi in narrazione collettiva. 


\section{Un "paesaggio della memoria"}

Quando si procede a ricostruire il profilo biografico di una comunità, oltre a una pressione della memoria sulla storiografia si innesca una pressione della storiografia sulla memoria, nel senso che l'evento al centro della narrazione diventa il prisma attraverso cui si interpretano e rielaborano i racconti individuali. Ogni particolare, così come ogni silenzio, omissione, o negazione, si carica di significato e va a comporre una narrazione autobiografica collettiva che può identificare la comunità, anche nei passaggi intergenerazionali delle memorie trasmesse $o$ taciute. È dunque possibile parlare di un "paesaggio della memoria" che rappresenta un patrimonio e una ricchezza per la comunità che abita in quel luogo e che ne custodisce l'identità più autentica (a patto che questa identità riesca però a dialogare con altre identità, dimostrando dunque anche capacità di accoglienza e ospitalità). Questo binomio tra ricerca e azione, utilizzando tecniche di racconti biografici, si connota anche come un'esplorazione e una valorizzazione del patrimonio memoriale dei luoghi che costituiscono lo sfondo delle storie e dei dispositivi memoriali, permettendo - a chi li interpreta e li rielabora - azioni partecipate che favoriscono l'integrazione sociale, proprio grazie alla riappropriazione del passato, al riconoscimento, al dialogo, allo scambio.

La territorialità delle memorie permette infatti di evidenziare i legami tra gli eventi storici e certi luoghi geografici particolari, i ricordi che ogni comunità ha rielaborato e trasmesso alle generazioni successive, portando così a delineare e produrre, se adeguatamente esplorata e interrogata, una "biografia di comunità". In questa proposta di ricerca-azione, però, il ruolo dei racconti autobiografici, diventati testi scritti e "documenti", è quello di permettere di ricostruire un quadro composito, plurimo, a volte anche conflittuale, ma indirizzato a una restituzione attiva della rielaborazione del passato e degli eventi oggetti di indagine. Una visione plurima e multifocale dell'identità collettiva di quella comunità che resta un oggetto di narrazione, "conservazione" di memorie individuali, le quali solo alla fine del processo di interpretazione vedono confluire i propri profili biografici in un profilo più generale, quello appunto di una "biografia della comunità".

\section{Tra storia e memoria, tra ricerca e didattica}

"Biografia di comunità" dunque si propone come una strumentazione potente ed efficace nell'ambito di ricerche storico-memoriali, perché assegna al ricercatore proprio il ruolo di interprete delle tracce fisiche e memoriali di una comunità in un dato luogo. E restituire una narrazione biografica collettiva significa affronta- 
re criticamente il rapporto con il passato che si esprime attraverso le "produzioni" (anche scritte e/o artistiche) delle diverse generazioni, imparando a collocare tali testimonianze in un complesso e articolato paesaggio della memoria che ne ricostruisca le differenti "stratificazioni", delineando quasi una storia della memoria all'interno di un contesto che, solo, può essere affidato alla storia.

In questo senso, la "biografia di comunità" può rappresentare un ponte tra memoria e storia, ed è in questa prospettiva che la metodologia che proponiamo può diventare un interessante percorso di approfondimento per chi, operando all'interno degli istituti, si misura quotidianamente con i problemi della didattica della storia e con il rapporto delle giovani generazioni con il passato.

\section{Un caso di studio: la Capolo di Montecchio Emilia}

Questa ricerca-azione si sta svolgendo da due anni (2013-15) a Montecchio Emilia, un paese pedecollinare della Val d'Enza, zona al confine tra due province, Reggio Emilia e Parma. Il lavoro ha per oggetto di studio l'industrializzazione e le trasformazioni del territorio, la nascita di un distretto industriale incardinato su una fabbrica, la Capolo, e i cambiamenti che nel lavoro e nelle dinamiche di sviluppo territoriale e nel tessuto sociale si sono determinati. Un paese, Montecchio Emilia, che per la sua particolare posizione geografica - centrale sull'asse nord/sud della via Emilia, fra gli Appennini e la pianura, e sull'asse est/ovest, fra le due città di Reggio Emilia e Parma - ha conosciuto uno sviluppo industriale particolare, tutto incentrato sulla fabbrica Capolo, che ha determinato, con l'indotto, la nascita di un vero e proprio distretto industriale, anche se di dimensioni più ridotte rispetto alle realtà di Carpi e Sassuolo.

La storia di questa fabbrica, dunque, rappresenta in modo emblematico il lungo Novecento dell'industrializzazione a Montecchio e in tutta la Val d'Enza, per le trasformazioni che ha determinato nella comunità, nel tessuto sociale e nelle realtà produttive del comprensorio. Una storia di lunga durata del lavoro operaio, delle maestranze e del terziario che ha contribuito a creare, attorno alla Capolo, un tessuto produttivo molto variegato e vivace. Una storia anche delle trasformazioni portate a Montecchio e nel comprensorio dalla modernizzazione, dall'industrializzazione di inizio Novecento per arrivare fino alla globalizzazione, con conseguente trasformazione della Capolo in una multinazionale, come conseguenza dei processi di terziarizzazione e di finanziarizzazione dell'economia e del sistema produttivo.

Questa storia è legata indissolubilmente alla fabbrica, nata nel 1910 in una casa del popolo "bianca", per opera di sacerdoti e laici. Protagonisti tutti quanti di una 
sfida che partì da un patto e da un sodalizio umano, finanziario e di lungimiranza imprenditoriale. Di questi protagonisti citiamo solo i due più noti: don Alai, sacerdote fondatore, e Minardi Silvio, lattoniere, che mise il proprio sapere al servizio della fabbrica nascente. Furono loro a costituire il 2 ottobre 1910, davanti al notaio Del Rio, la Società anonima cooperativa cattolica, con un capitale sociale di 720 lire e un capitale di risorse umane e competenze notevole per il tempo (comprendeva, tra i fondatori, un impiegato, diversi possidenti terrieri e qualche artigiano calzolaio).

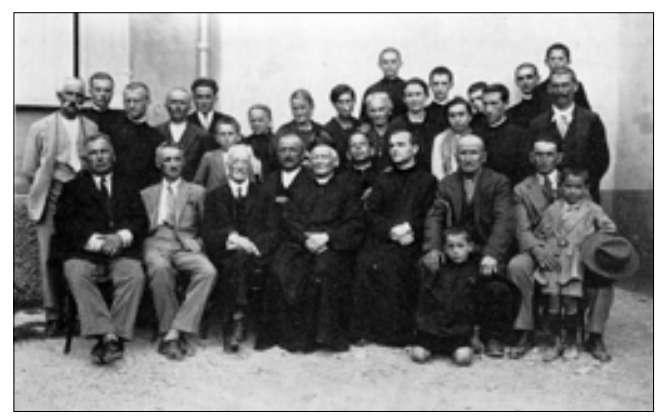

Don Alai e il gruppo di fondatori della Capolo.

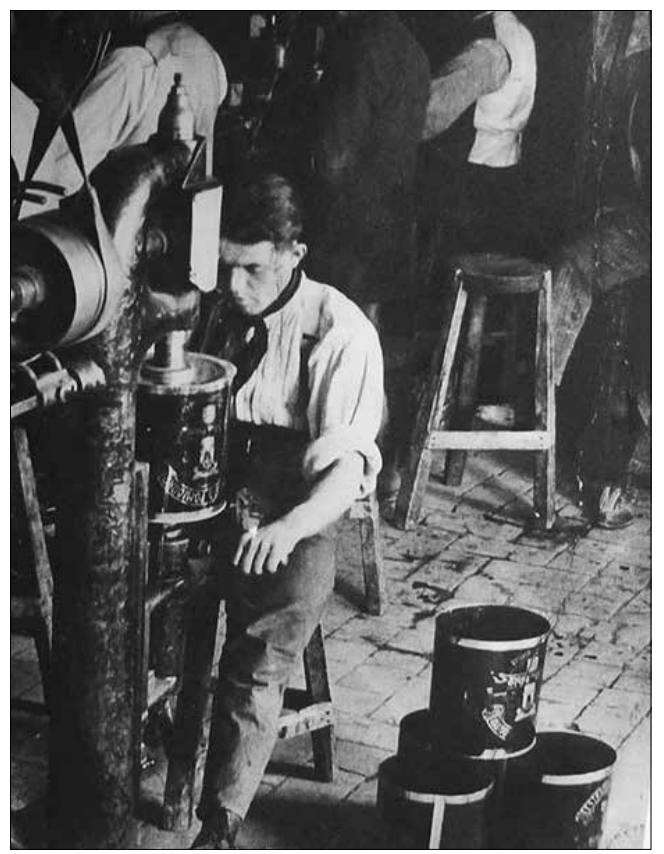

Il lavoro in officina.
La storia successiva fu quella di una fabbrica che si sviluppò producendo barattoli di latta e intrecciando la propria produzione con quella del pomodoro - tipica coltivazione agricola del parmense -, diventando così un prisma potente per leggere i processi storici dal boom economico fino al tempo attuale, in cui la Capolo è diventata una multinazionale.

Il quadro politico e socio-economico del territorio montecchiese alla fine dell'Ottocento presentava alcune caratteristiche interessanti per comprenderne l'evoluzione produttiva. Caratteristiche peraltro abbastanza comuni ad altri territori della nostra regione e dell'Italia di quel periodo storico. La posizione in zona pedecollinare e vicino al fiume Enza, ricco di depositi di sabbia e sassi - materiali allora importanti per l'edilizia -, la vicinanza della Via Emilia e la posizione centrale fra Reggio Emilia e Parma - molto favorevole agli scambi e ai commerci - facevano di Montecchio Emilia una cittadina abbastanza vi- 
vace e prosperosa, già a partire dagli ultimi decenni dell'Ottocento, benché l'agricoltura fosse ancora l'attività prioritaria della popolazione. Anche le caratteristiche morfologiche del territorio rappresentano bene questa sua centralità fra l'asse nord-sud e est-ovest. Montecchio appariva infatti circondata da un "reticolato" di case coloniche, di poderi, di filari, di coltivazioni, anche se il suo sistema agricolo non garantiva in quel periodo una produttività elevata, a causa della prevalenza della mezzadria che non permetteva, per varie ragioni, l'applicazione di tecniche innovative nella coltivazione dei campi e faceva ricorso invece a massicce e diffuse forme di sfruttamento della forza-lavoro di braccianti, fra cui molte donne e bambini. Non a caso, già dalla fine dell'Ottocento e poi ancora nei primi decenni del Novecento, da queste zone si generò un forte movimento migratorio verso la Francia e l'Europa del nord.

L'associazionismo operaio di fine Ottocento e poi il cooperativismo, molto vivaci e attivi in tutta la zona del reggiano, in questo territorio assunsero un indirizzo prevalentemente cattolico, al contrario di molte altre zone del reggiano in cui il "prampolinismo" - cioè la particolare declinazione del socialismo proposta da Camillo Prampolini - risultava prevalente. Si tratta di fenomeni importanti per capire la realtà storica della regione in quei decenni di inizio Novecento, perché si configurarono risposte importanti al problema della mancanza del lavoro e dello sfruttamento della manodopera, con la ricerca di forme di tutela per gli operai e i braccianti che ovviassero all'assenza di legislazione sociale e previdenziale.

La nascita di una fabbrica come la Capolo cambiò lo scenario, portando un investimento di risorse economiche e umane nella produzione della latta e trasformando questa attività nella produzione di barattoli per conserve e pomodori. L'inizio e poi l'evoluzione dell'attività produttiva della fabbrica è molto interessante anche come caso di studio, su scala locale, di fenomeni storici e processi socioeconomici che interessarono numerose zone del nostro paese. Infatti la dirigenza e le maestranze della Capolo seppero affrontare, con coraggio e impegno, l'impatto forte e devastante della Prima guerra mondiale (molti operai, compresi i più esperti, dovettero partire per il fronte), dimostrando che l'intelligenza, l'estro e l'intuito, che avevano contribuito a farla nascere, potevano essere ancora le leve per un ulteriore sviluppo.

Negli anni Venti, la Capolo dovette misurarsi prima con l'avvento del fascismo e poi con un regime pienamente consolidato, con il quale non intrattenne rapporti idilliaci, subendo per questo diversi contraccolpi e conseguenze negative. Tuttavia, questo periodo di difficoltà non riuscì a deprimere del tutto l'assetto produttivo, ancora una volta grazie alla lungimiranza e alla capacità imprenditoriale della dirigenza e degli operai, fra cui comunque si era instaurata una significativa 
solidarietà e una vera e propria alleanza per il miglioramento della produzione. Le riflessioni storiche e didattiche sulle dinamiche memoriali nel contesto montecchiese hanno portato a concepire e proporre diverse modalità di raccolta di biografie, proprio per la lunga durata del processo di industrializzazione studiato, per la molteplicità dei protagonisti coinvolti e per la forte valenza simbolica che il luogo di questa fabbrica assume per la comunità e il territorio. Dunque, alle metodologie ricorrenti e consolidate, sono state scelte, in questa ricerca-azione, anche metodologie fortemente interattive e laboratoriali, quali l'esplorazione di diversi luoghi significativi del territorio, il censimento di lavoratori della Capolo attraverso legami e memorie familiari, le interviste reali ai testimoni con registrazione e documentazione del racconto biografico, e le interviste impossibili a protagonisti del passato, ricostruite dagli studenti e da loro reinterpretate con abiti, oggetti e ambientazioni proprie del periodo storico esaminato e della comunità di cui erano espressione.

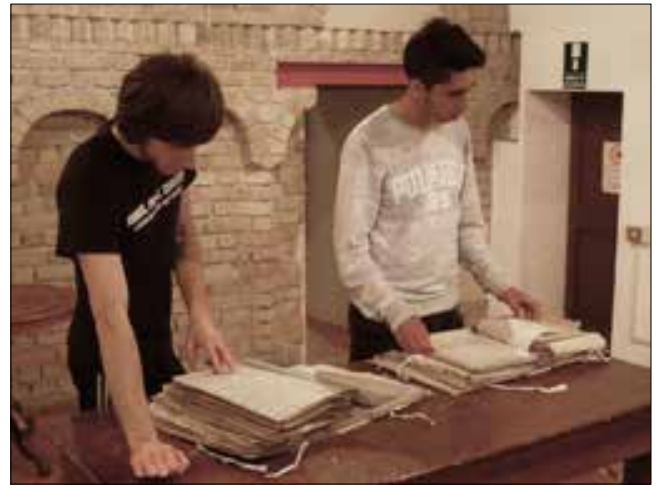

Ricerche in archivio.

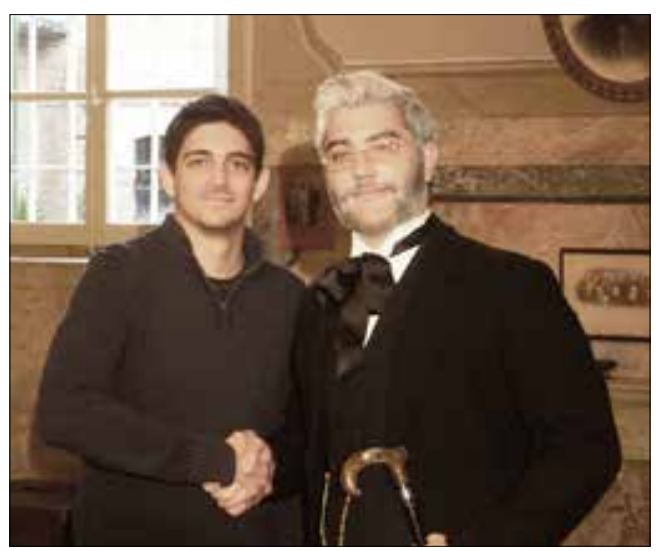

Interviste immaginarie.
Le diverse metodologie didattiche utilizzate per raccogliere biografie (interviste, visite guidate ai luoghi, resoconti memoriali di protagonisti dell'attività produttiva della fabbrica) hanno permesso di documentare, archiviare e conservare un materiale molto interessante e ricco di fonti e documenti di varia natura. L'utilizzo di questo materiale, raccolto con la strumentazione del metodo biografico, deve supportare la ricostruzione storica dell'industrializzazione attraverso l'esplorazione e l'osservazione dei luoghi, tramite lo studio e l'analisi di archivi e documenti vari, ma anche, o soprattutto, attraverso le testimonianze dei protagonisti della produzione industriale: gli operai e gli impiegati che alla Capolo hanno conosciuto una fondamentale esperienza lavorativa e di vita. 